\title{
AN EXAMPLE IN THE GRADIENT THEORY OF PHASE TRANSITIONS
}

\author{
CAmillo De Lellis ${ }^{1}$
}

\begin{abstract}
We prove by giving an example that when $n \geq 3$ the asymptotic behavior of functionals $\int_{\Omega} \varepsilon\left|\nabla^{2} u\right|^{2}+\left(1-|\nabla u|^{2}\right)^{2} / \varepsilon$ is quite different with respect to the planar case. In particular we show that the one-dimensional ansatz due to Aviles and Giga in the planar case (see [2]) is no longer true in higher dimensions.
\end{abstract}

Mathematics Subject Classification. 49J45, 74G65, 76M30.

Received December 4, 2001.

\section{INTRODUCTION}

This paper is devoted to the study of the asymptotic behavior of functionals

$$
F_{\varepsilon}^{\Omega}(u):=\int_{\Omega}\left(\varepsilon\left|\nabla^{2} u\right|^{2}+\frac{\left(1-|\nabla u|^{2}\right)^{2}}{\varepsilon}\right) \quad \Omega \subset \mathbf{R}^{n}
$$

as $\varepsilon \downarrow 0$, where $u$ maps $\Omega$ into $\mathbf{R}$. This problem was raised by Aviles and Giga in [2] in connection with the mathematical theory of liquid crystals and more recently by Gioia and Ortiz in [9] for modeling the behavior of thin film blisters. Recently many authors have studied the planar case giving strong evidences that, as conjectured by Aviles and Giga in [2], the sequence $\left(F_{\varepsilon}\right) \Gamma$-converge (in the strong topology of $W^{1,3}$ : see [1] for a discussion of such a choice and a rigorous setting) to the functional

$$
F_{\infty}^{\Omega}(u):= \begin{cases}\frac{1}{3} \int_{J_{\nabla v}}\left|\nabla u^{+}-\nabla u^{-}\right|^{3} \mathrm{~d} \mathcal{H}^{n-1} & \text { if }|\nabla u|=1, u \in W^{1, \infty} \\ +\infty & \text { otherwise. }\end{cases}
$$

Here $J_{\nabla u}$ denotes the set of points where $\nabla u$ has a jump and $\left|\nabla u^{+}-\nabla u^{-}\right|$is the amount of this jump. Of course the first line of the previous definition makes sense only for particular choices of $u$, such as piecewise $C^{1}$. For a rigorous setting the reader should think about a suitable function space $S$ which contains piecewise $C^{1}$ functions and on which we can give a precise meaning to the above integral (for example a natural choice would be $\{u \mid \nabla u \in B V\}$; however this space turns out not to be the natural one: we refer again to [1] for a discussion of this topic).

Partial results in proving Aviles and Giga's conjecture (i.e. compactness of minimizers of $F_{\varepsilon}^{\Omega}$, estimates from below on $F_{\varepsilon}^{\Omega}\left(u_{\varepsilon}\right)$ and a suitable weak formulation for the problem of minimizing $F$ subject to some boundary conditions) can be found in $[1,3,5-8]$.

Keywords and phrases: Phase transitions, Г-convergence, asymptotic analysis, singular perturbation, Ginzburg-Landau.

1 Scuola Normale Superiore, P.zza dei Cavalieri 7, 56100 Pisa, Italy; e-mail: delellis@cibs.sns.it 
In their first work Aviles and Giga based their conjecture on the following ansatz (which they made in the case $n=2)$ :

Conjecture 1.1. Let us choose a map $w: \Omega \rightarrow \mathbf{R}$ (with $\Omega \subset \mathbf{R}^{n}$ bounded open set containing 0 ) such that:

(a) $w$ is Lipschitz and satisfies the eikonal equation $|\nabla w|=1$;

(b) $\nabla w$ is constant in $\left\{x_{1}<0\right\}$ and in $\left\{x_{1}>0\right\}$.

Let us define $E:=\inf \left\{\liminf _{\varepsilon} F_{\varepsilon}^{\Omega}\left(u_{\varepsilon}\right):\left\|u_{\varepsilon}-w\right\|_{W^{1,3}} \rightarrow 0\right\}$. Then there exists a family of functions $w_{\varepsilon}$ such that:

(i) the component of $\nabla w_{\varepsilon}$ perpendicular to $(1,0, \ldots, 0)$ is constant;

(ii) $w_{\varepsilon} \rightarrow w$ in $W^{1,3}$;

(iii) $\lim F_{\varepsilon}^{\Omega}\left(w_{\varepsilon}\right)=E$.

This ansatz has been proved by Jin and Kohn in [8] for $n=2$. It reduces the problem of finding $E$ to a one dimensional problem in the calculus of variations which can be explicitly solved. This analysis leads to the result $E=F_{\infty}^{\Omega}(w)$, which means that at $w$ the $\Gamma$-limit of $F_{\varepsilon}^{\Omega}$ exists and coincides with $F_{\infty}^{\Omega}(w)$. With a standard cut and paste argument (see [4]) it can be proved that the same happens for every $w$ which is piecewise affine. In the next section we will prove the following theorem:

Theorem 1.2. Let $u$ be the function $u\left(x_{1}, x_{2}, x_{3}\right)=\left|x_{3}\right|$ and $C$ the cylinder $\left\{\left|x_{1}\right|^{2}+\left|x_{2}\right|^{2}<1\right\}$. Then there exists $\left(u_{k}\right)$ such that:

(a) every $u_{k}$ is piecewise affine (being the union of a finite number of affine pieces) and satisfies the eikonal equation;

(b) $\lim _{k} F_{\infty}^{C}\left(u_{k}\right)<F_{\infty}^{C}(u)$;

(c) $u_{k} \rightarrow u$ strongly in $W^{1, p}$ for every $p<\infty$.

The proof can be easily generalized to every $n \geq 3$. As an easy corollary we get that the one-dimensional ansatz fails for $n \geq 3$. Moreover this failure means that $F$ cannot be the $\Gamma$-limit of $F_{\varepsilon}^{\Omega}$ for $n \geq 3$.

Corollary 1.3. The one-dimensional ansatz is not true for $n \geq 3$.

Proof. As already observed, being every $u_{k}$ piecewise affine, there is a family of functions $u_{k, \varepsilon}$ such that $u_{k, \varepsilon}$ converge to $u_{k}$ in $W^{1, p}$ (for every $p<\infty$ ) and $\lim _{\varepsilon} F_{\varepsilon}^{C}\left(u_{k, \varepsilon}\right)=F_{\infty}^{C}\left(u_{k}\right)$. A standard diagonal argument gives a sequence $\left(u_{k, \varepsilon(k)}\right)$ strongly converging to $u$ in $W^{1, p}$ such that $\lim _{k} F_{\varepsilon(k)}^{C}\left(u_{k, \varepsilon(k)}\right)<F_{\infty}^{C}(u)$.

\section{THE EXAMPLE}

In this section we prove Theorem 1.2. First of all we recall the following fact:

(Curl) If $v: \mathbf{R}^{n} \rightarrow \mathbf{R}^{n}$ is a piecewise constant vector field, then $v$ is a gradient if and only if for every hyperplane of discontinuity $\pi$ the right trace and the left trace of $v$ have same component parallel to $\pi$.

The building block of the construction of Theorem 1.2 is the following vector field, depending on a parameter $\phi \in(0, \pi / 2)$. First of all we fix in $\mathbf{R}^{3}$ a system of cylindrical coordinates $(r, \theta, z)$ and then we call $A$ the cone given by $\{z>0, r<1,(1-r)>z \tan \phi\}$ and $A^{\prime}$ the reflection of $A$ with respect to the plane $\{z=0\}$. Hence we put

$$
\begin{array}{lr}
v(r, \theta, z)=(0,0,1) & \text { if } z>0 \text { and }(r, \theta, z) \notin A \\
v(r, \theta, z)=(\sin (2 \phi), \theta+\pi, \cos (2 \phi)) & \text { if } z>0 \text { and } z \in A \\
v(r, \theta, z)=(0,0,-1) & \text { if } z<0 \text { and }(r, \theta, z) \notin A^{\prime} \\
v(r, \theta, z)=(\sin (2 \phi), \theta+\pi,-\cos (2 \phi)) & \text { if } z<0 \text { and } z \in A^{\prime} .
\end{array}
$$


It is easy to see that $v$ maps every plane $\{\theta=\alpha\} \cup\{\theta=\alpha+\pi\}$ into itself. Moreover the restrictions of $v$ to these planes all look like as in the following picture

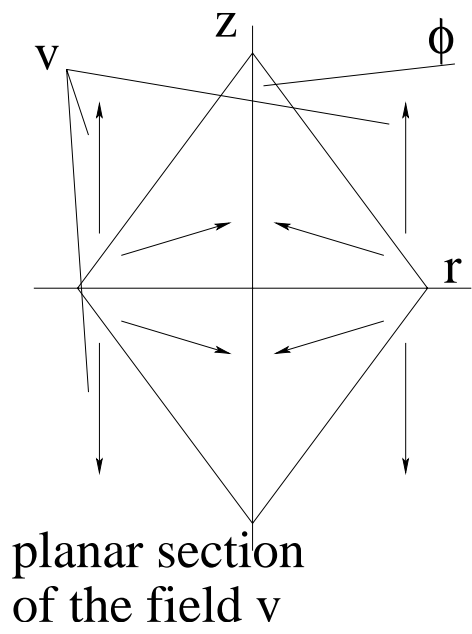

Lemma 2.1. The vector field $v$ is the gradient of a function $w$. Moreover there is a sequence of piecewise affine functions $w_{k}$ such that:

(a) $w_{k} \rightarrow w$ strongly in $W_{\text {loc }}^{1, p}$ for every $p$;

(b) $F_{\infty}^{\Omega}\left(w_{k}\right) \rightarrow F_{\infty}^{\Omega}(w)$ for every open set $\Omega \subset \subset \mathbf{R}^{3}$.

Proof. We consider the restriction of $v$ to the plane $P:=\{\theta=0\} \cup\{\theta=\pi\}$. As already noticed $v$ maps this plane into itself. Moreover its restriction to it satisfies condition (Curl), hence on $P v$ is the gradient of a scalar function $w$. Moreover we can find such a $w$ so that it is identically zero on the line $\{z=0\} \cap P$. Hence $w$ is symmetric with respect to the $z$ axis and so we can extend $w$ to the whole three-dimensional space so to build a cylindrically symmetric function. It is easy to check that the gradient of such a function is equal to $v$.

We call this function $w$ as well and we will prove that it satisfies conditions (a) and (b) written above.

(a) Our goal is approximating $v$ with piecewise constant gradient fields. First of all we do it in the upper half-space $\{z>0\}$. For every $n$ we take a regular $n$-agon $B_{n}$ which is inscribed to the circle of radius 1 and lies on the plane $\{z=0\}$. The vertices of this $n$-agon are given by $V_{i}:=(1,2 i \pi / n, 0)$.

Hence we construct the pyramid $A^{n}$ with vertex $V:=(0,0, \cot \phi)$ and base $B_{n}$. In the pyramid we identify $n$ different regions $A_{1}^{n}, \ldots, A_{n}^{n}$, where every $A_{i}^{n}$ is given by the tetrahedron with vertices $(0,0,0), V, V_{i}, V_{i+1}$. After this we put $v_{n}$ equal to $(0,0,1)$ outside $A^{n}$ and in every $A_{i}^{n}$ we put

$$
v_{n}(r, \theta, z) \equiv(\sin 2 \phi, \pi+(2 i+1) \pi / n, \cos 2 \phi) .
$$

It is easy to see that $v_{n}$ satisfies condition (Curl), hence it is the gradient of some function $w_{n}$. Moreover we can choose $w_{n}$ in such a way that it is identically 0 on $\{z=0\}$. Then we extend $w_{n}$ to the lower half space $\{z<0\}$ just by imposing $w_{n}(r, \theta,-z)=w_{n}(r, \theta, z)$. It is not difficult to see that $\nabla w_{n}$ converges strongly to $\nabla w$ in $L_{l o c}^{p}$ for every $p$.

(b) Now we check that the previous construction satisfies also the second condition of the lemma. We fix an open set $\Omega \subset \subset \mathbf{R}^{3}$ and we observe that both $w_{k}$ and $w$ satisfy the eikonal equation in $\Omega$. Moreover we call $L_{i}^{n}$ the triangle with vertices $V, V_{i}, V_{i+1}$ and $L^{n}$ the union of $L_{i}^{n}$ (so $L^{n}$ is the "lateral surface" of the pyramid $\left.A^{n}\right)$. Finally we denote by $L$ the lateral surface of the cone $A$, i.e. the set $\{(1-r)=z \tan \phi\}$.

(i) The amount of jump of $v_{n}$ (i.e. $\left.\left|v_{n}^{+}-v_{n}^{-}\right|\right)$on $L^{n}$ is constant and equal to the value of $\left|v^{+}-v^{-}\right|$on $L$. Moreover the area of $L^{n}$ is converging to the area of $L$. The same happens on the symmetric sets in the lower half-space $\{z<0\}$. 
(ii) Let us call $B$ the base of the cone. The right and left traces of $v_{n}$ coincides with those of $v$ on $B_{n} \cup(\{z=$ $0\} \backslash B)$. Moreover the area of $B \backslash B_{n}$ is converging to zero.

(iii) The vector fields $v_{n}$ are discontinuous also on the triangles $T_{i}^{n}$ joining $V,(0,0,0)$ and $V_{i}$ (and on the symmetric triangles lying on $\{z<0\}$ ). The amount of jump of $v_{n}$ on each of these triangles is given by

$$
\left|v_{n}^{+}-v_{n}^{-}\right|=2 \sin (\pi / n) .
$$

Moreover the area of everyone is given by $(\cot \phi) / 2$. Hence

$$
\int_{\cup_{i} T_{i}^{n}}\left|v_{n}^{+}-v_{n}^{-}\right|^{3} \mathrm{~d} \mathcal{H}^{2}=4 n \cot \phi \sin ^{3} \pi / n .
$$

The right hand side goes to zero as $n \rightarrow \infty$ and this completes the proof.

Proof of Theorem 1.2. First of all we pass from the cartesian coordinates of the statement to the cylindrical coordinates $(r, \theta, z)$ given by $x_{3}=z, x_{1}=r \cos \theta, x_{2}=r \sin \theta$ (and sometimes we will denote the elements of $\mathbf{R}^{3}$ with $(y, z)$, where $y \in \mathbf{R}^{2}$ and $z \in \mathbf{R}$ ).

We take $w$ as in the previous lemma. First of all let us compute $F_{\infty}^{C}(w)$ where $C$ is the cylinder $\{r<1\}$. As in the previous proof we call $L$ the lateral surface of the cone, that is the set $\{r-1=z \tan \phi\}$. The value of $\left|\nabla w^{+}-\nabla w^{-}\right|$on the surface $L$ is given by $2 \sin \phi$ and the area of $L$ is given by $\pi / \sin \phi$ : the same happens for the symmetric of $L$ lying on the half-space $\{z<0\}$. On the base of the cylinder we have $\left|\nabla w^{+}-\nabla w^{-}\right|=2|\cos 2 \phi|$. Hence

$$
a(\phi):=F_{\infty}^{C}(u)-F_{\infty}^{C}(w)=\frac{\pi}{3}\left[8-8 \cos ^{3} 2 \phi-16 \sin ^{2} \phi\right]
$$

and it can be easily checked that for $\phi$ close enough to zero, $a(\phi)$ is positive.

Therefore let us fix an $\alpha$ for which $a(\alpha)>0$ and let us agree that $w$ is constructed as in the previous lemma by choosing $\phi=\alpha$. Given $\rho>0$ and $x \in \mathbf{R}^{2}$ we define $w_{x, \rho}$ in the cylinder $C_{x, \rho}:=\{(y, z):|y-x| \leq \rho\} \subset \mathbf{R}^{3}$ as $w_{x, \rho}(y, z)=\rho w((y-x) / \rho, z / \rho)$. It is easy to see that

$$
F_{\infty}^{C_{x, \rho}}(u)-F_{\infty}^{C_{x, \rho}}\left(w_{x, \rho}\right)=a(\alpha) \rho^{2} .
$$

Let us fix $\varepsilon$ and take $\rho$ such that $\rho \cot \alpha<\varepsilon$. Thanks to Besicovitch Covering lemma we can cover $\mathcal{H}^{2}$ almost all $D:=\{z=0, r \leq 1\}$ with a disjoint countable family of closed discs $D_{i}$ such that every $D_{i}$ has radius $r_{i}<\rho$, center $x_{i}$ and is contained in $D$. We construct $u_{\varepsilon}$ by putting $u_{\varepsilon} \equiv w_{x_{i}, \rho_{i}}$ in the cylinder $C_{x_{i}, \rho_{i}}$.

Since $\nabla u_{\varepsilon}$ coincides with $\nabla u$ in $\{z \geq \varepsilon\}$ and satisfies the eikonal equation, it is easy to see that $u_{\varepsilon} \rightarrow u$ locally in the strong topology of $W^{1, p}$. Moreover equation (2) implies that

$$
F_{\infty}^{C}(u)-F_{\infty}^{C}\left(u_{\varepsilon}\right)=\sum_{i} a(\alpha) r_{i}^{2}=a(\alpha)
$$

At this point, using the previous lemma we can approximate the function $u_{\varepsilon}$ in the cylinders $C_{x_{i}, \rho_{i}}$ with piecewise affine functions in such a way that their traces coincide with the trace of $u_{\varepsilon}$ on the boundary of $C_{x_{i}, \rho_{i}}$. Using standard diagonal arguments for every $\varepsilon$ we can find a sequence of piecewise affine functions $u_{\varepsilon}^{k}$ which converge in $W^{1, p}$ to $u_{\varepsilon}$ and such that $F_{\infty}^{C}\left(u_{\varepsilon}^{k}\right) \rightarrow F_{\infty}^{C}\left(u_{\varepsilon}\right)$. Moreover, again using diagonal arguments, we can construct the sequence $u_{\varepsilon}^{k}$ so that each one is a finite union of affine pieces.

Finally, one last diagonal argument, gives a sequence $\tilde{u}_{k}$ suh that:

(a) $\tilde{u}_{k}$ is a finite union of affine pieces;

(b) $\lim _{k} F_{\infty}^{C}\left(\tilde{u}_{k}\right)<F_{\infty}^{C}(u)$;

(c) $\tilde{u}_{k} \rightarrow u$ strongly in $W^{1, p}$ for every $p<\infty$. 


\section{REFERENCES}

[1] L. Ambrosio, C. De Lellis and C. Mantegazza, Line energies for gradient vector fields in the plane. Calc. Var. Partial Differential Equations 9 (1999) 327-355.

[2] P. Aviles and Y. Giga, A mathematical problem related to the physical theory of liquid crystal configurations. Proc. Centre Math. Anal. Austral. Nat. Univ. 12 (1987) 1-16.

[3] P. Aviles and Y. Giga, On lower semicontinuity of a defect energy obtained by a singular limit of the Ginzburg-Landau type energy for gradient fields. Proc. Roy. Soc. Edinburgh Sect. A 129 (1999) 1-17.

[4] C. De Lellis, Energie di linea per campi di gradienti, Ba. D. Thesis. University of Pisa (1999).

[5] A. De Simone, R.W. Kohn, S. Müller and F. Otto, A compactness result in the gradient theory of phase transition. Proc. Roy. Soc. Edinburgh Sect. A 131 (2001) 833-844.

[6] P.-E. Jabin and B. Perthame, Compactness in Ginzburg-Landau energy by kinetic averaging. Comm. Pure Appl. Math. 54 (2001) 1096-1109.

[7] W. Jin, Singular perturbation and the energy of folds, Ph.D. Thesis. Courant Insitute, New York (1999).

[8] W. Jin and R.V. Kohn, Singular perturbation and the energy of folds. J. Nonlinear Sci. 10 (2000) 355-390.

[9] M. Ortiz and G. Gioia, The morphology and folding patterns of buckling driven thin-film blisters. J. Mech. Phys. Solids 42 (1994) 531-559. 